\title{
Validation and Test Cases for a Free Surface SPH Model
}

\author{
Louis Goffin, Sébastien Erpicum, Benjamin J. Dewals, \\ Michel Pirotton and Pierre Archambeau
}

\section{Introduction}

Numerical methods in hydraulics can be separated into two categories. On the one hand we have grid-based methods and on the other hand we have meshfree methods [17]. In the meshfree methods category, SPH is one the most used. Smoothed-particle hydrodynamics was first introduced in the field of astrophysics by Gingold and Monaghan [6] and Lucy [20] in 1977 but is now used in hydraulics [3, 12, 23], structure dynamics [1], solid mechanics [26], etc.

Nowadays, the method is still under development but a wide range of problems can be modeled: it is used in many fields of application and implemented in the well-known open-source program SPHysics [8, 9]. The goal of this paper is to identify some undesired effects linked to the SPH method as well as to give some validation benchmark. We will first introduce the SPH method in the frame of open-channel quasi-incompressible flows. Then, various implementation aspects that developers can encounter will be presented. The main part of this paper will focus on validation test cases. They will highlight some issues linked to the SPH method and the answers given recently. Finally, a brand-new test case will be described.

\section{The SPH Method}

The main principle of the SPH method, as explained in [21], is based on the approximation of a function

L. Goffin $(\bowtie)$ - S. Erpicum · B.J. Dewals · M. Pirotton · P. Archambeau

Hydraulics in Environmental and Civil Engineering (HECE), University of Liege (ULg),

Allée de la Découverte 9 (B52/3), B-4000 Liège, Belgium

e-mail: 1.goffin@ulg.ac.be 


$$
\langle f(\mathbf{x})\rangle=\int_{\Omega} f\left(\mathbf{x}^{\prime}\right) W\left(\mathbf{x}-\mathbf{x}^{\prime}, h\right) d \mathbf{x}^{\prime}
$$

where $f$ is a function, $W$ a smoothing function, $\Omega$ the domain where $f$ is defined, and $h$ the smoothing length. The particle approximation can be applied to Eq. (1):

$$
\left\langle f\left(\mathbf{x}_{a}\right)\right\rangle=\sum_{b=1}^{N} \frac{m_{b}}{\rho_{b}} f\left(\mathbf{x}_{b}\right) W_{a b}
$$

where the subscripts $a$ and $b$ refer, respectively, to the current particle and the neighbor particles, $N$ is the number of neighbors, $m$ is the mass of a particle, $\rho$ its density, and $W_{a b}=W\left(\mathbf{x}_{a}-\mathbf{x}_{b}, h\right)$.

For more details about the establishment of this formalism, the reader can refer to [18] or [7].

\subsection{Equations and Smoothing Functions}

Newtonian fluids are ruled by Navier-Stokes equations. When the SPH formalism is applied to these equations, we obtain the density continuity

$$
\frac{D \rho_{a}}{D t}=\sum_{b=1}^{N} m_{b} \mathbf{u}_{a b} \nabla_{a} W_{a b}
$$

where the speed $\mathbf{u}_{a b}=\mathbf{u}_{a}-\mathbf{u}_{b}$, and a derived form of the conservation of momentum

$$
\frac{D \mathbf{u}_{a}}{D t}=-\sum_{b=1}^{N} m_{b}\left(\frac{p_{b}}{\rho_{b}^{2}}+\frac{p_{a}}{\rho_{a}^{2}}+\Pi_{a b}\right) \nabla_{a} W_{a b}+\mathbf{F}
$$

where $p$ is the pressure, $\Pi_{a b}$ an artificial viscosity term, and $\mathbf{F}$ the body forces.

The smoothing function $W$ in Eqs. (3) and (4) are most often a cubic spline [24], a Gaussian [6] or a quadratic function [13]. In this paper, we will use exclusively a cubic spline.

\subsection{Time Integration Scheme}

The time integration scheme used in this work is a Runge-Kutta 22 scheme (abbreviated RK22): 


$$
\begin{aligned}
y_{i+1} & =y_{i}+h\left((1-\theta) k_{1}+\theta k_{2}\right) \quad ; \quad k_{1}=f\left(t_{i}, y_{i}\right) \quad ; \\
k_{2} & =f\left(t_{i}+\frac{1}{2 \theta} h, y_{i}+\frac{1}{2 \theta} h k_{1}\right)
\end{aligned}
$$

with $\theta=0.5$ and where $t_{i+1}=t_{i}+h$ and $f\left(t_{i}, y_{i}\right)=y_{i}^{\prime}$. Another RK22 scheme is obtained with $\theta=1$ and the Euler scheme is obtained for $\theta=0$. These three integration schemes are compared for a dam break on dry bed in Sect. 3.

In the SPH literature, the Leapfrog scheme is often used for its stability [29].

\subsection{Some Implementation Aspects}

In order to implement a SPH code, the developer must be aware of some particularities of the method.

As the smoothing functions are most often supported on a compact, the sum in the Eqs. (3) and (4) can be limited to some particles within a given distance to the computed particle (smoothing length). In order to determine these neighbors, there are basically three possibilities [18]: (a) all-pair search algorithm, (b) linked list algorithm, and (c) tree search algorithm. The second procedure is the fastest one as its speed is of the order of $N$. However, if a variable smoothing length in space is used, the third algorithm is more appropriate.

Concerning the smoothing length, it can be variable in time and/or in space. $s$ being the initial spacing between the particles, the initial smoothing length $h_{0}=1.2 \mathrm{~s}$ but it can range from 1 to $2 \mathrm{~s}$. In this work, we chose a coefficient of 1.2 which is a good compromise between the computational efficiency and the number of particles included in a support domain. In order to deal with the variable distance between particles, the smoothing length can be updated at every time step [24]:

$$
h=h_{0}\left(\frac{\rho_{0}}{\rho_{m}}\right)^{d}
$$

where $d$ is the number of dimensions and $\rho_{m}$ the mean density. It is obvious that the density is not likely to be homogenous in the whole domain. A possibility would be to have an adaptive smoothing length in space. However, such a method requires more precautions as Newton's third law might be violated as highlighted in [25].

The speed of sound plays an important role in any numerical fluid simulation. In fact, it rules the time step. In the case of free surface flows Monaghan [22] shows that the initial speed of sound can be taken at $c_{0}=10 \sqrt{g H}$ where $H$ is the maximum depth. It allows larger time steps and thus a quicker resolution. However, it introduces an artificial compressibility.

Boundaries can be modeled using different methods, including (a) ghost particles [26], (b) repulsive particles [22], and (c) dynamic particles [2]. For this work, dynamic particles were used for their flexibility and ease of use. We can model 
complex shapes, thanks to this kind of particles. However, there is no consensus in the SPH community about boundary conditions. It is one of the grand challenges on the SPH European Research Interest Community (SPHERIC).

Concerning initialization, particles can be set in a Cartesian grid or in a staggered way. Once their position is chosen, their pressure can be calculated. In most situations, the initial pressure is taken to the hydrostatic pressure. The pressure is not a direct variable in an SPH simulation but is related to the density. This link can be made, thanks to

$$
p=B\left(\left(\frac{\rho}{\rho_{0}}\right)^{\gamma}-1\right)
$$

where $B=c_{0}^{2} \rho_{0} / \gamma$ and $\gamma$ is set around 7. Equation (7) was introduced by Monaghan [22] and is based on Tait's equation [4] which is based on experimental measures. According to Violeau [28], the mass of a particle is set according to its volume $V_{a}=s^{d}$ and its density: $m_{a}=V_{a} \rho_{a}$. Considering hydrostatic pressure at the beginning of the simulation leads to particles having different masses. This mass, however, remains unchanged during the whole simulation.

\section{Test Cases and Undesired Effects}

In order to be validated, a code must pass some test cases. These test cases allow the developer to point his possible mistakes but also to realize the weakness of a method. This section will focus on some test cases and the conclusions that can be made. The characteristics of every test case are given in Table 1 in the order in which each test case appears.

Table 1 Characteristics of each test case

\begin{tabular}{l|l|l|l|l|l}
\hline & TC 1 & TC 2 & TC 3 & TC 4 & TC 5 \\
\hline $\mathrm{Nb}$ fixed part. $[-]$ & 6196 & 9894 & 60402 & 236390 & 73614 \\
\hline $\mathrm{Nb}$ mob. part. $[-]$ & 35836 & 47175 & 123750 & $668160-767691$ & $400200-504531$ \\
\hline $\mathrm{h}_{0}[\mathrm{~m}]$ & 0.024 & 0.024 & 0.024 & 0.012 & 0.024 \\
\hline$\alpha[-]$ & 0.05 & $0.2-0.01$ & 0.5 & 0.05 & 0.01 \\
\hline Simul. time [s] & 5 & 7 & 2 & 5 & 5 \\
\hline
\end{tabular}




\subsection{Tank of Still Water}

This test case is simply a box that contains an amount of water with free surface. The particles are initially set with hydrostatic pressure and without velocity. It is supposed that the particles will not move and will keep their initial pressure. The results are not as expected. Figure 1 shows the pressure distribution in the fluid at different times of the simulation. Tests were made with a correction of the kernel gradient but no improvements were noticed.

In order to explain this phenomenon, let us take the problem in another way: how could we set the particles in order to have an initial equilibrium? The answer can be obtained by solving a system

$$
A m=b
$$

where $m$ is a vector that contains the masses of the particles, $b$ is a vector that contains the body forces that act on a particle and

$$
A_{a, b}=-\left(\frac{p_{b}}{\rho_{b}^{2}}+\frac{p_{a}}{\rho_{a}^{2}}\right) \nabla_{a} W_{a b}
$$

System (8) indicates that the variation of velocity of a particle should be equal to zero according to Eq. (4). The boundary conditions can be handled by adding a reaction force (that is determined iteratively) to the boundary particles.

Matrix $A$ in system (8) is antisymmetric which makes it singular for an odd number of particles. This leads to impossibility to solve the system. We can conclude that an initial equilibrium can be found only for an even number of particles.
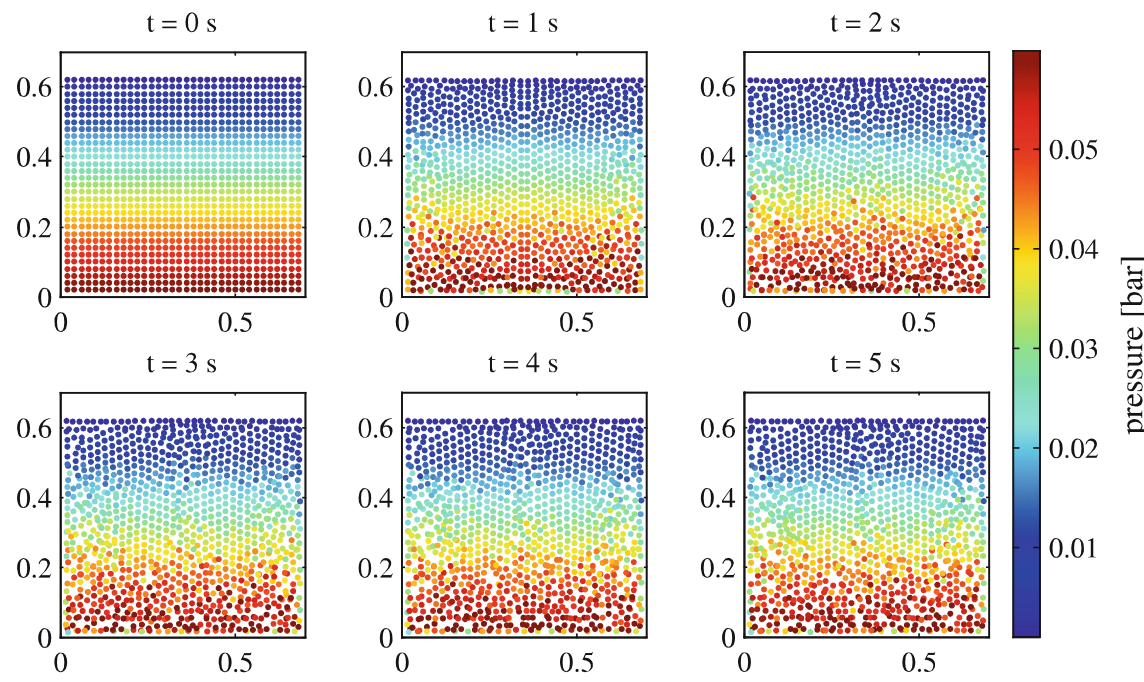

Fig. 1 Even if initially the pressure is hydrostatic, it is becoming less uniform with time 
Even when this condition is satisfied, the masses are not linearly distributed and some oscillations appear. The reader may find more information and examples in [7].

\subsection{Spinning Tank}

A spinning cylindrical tank of water presents a free surface that has the equation

$$
z(r)=\frac{r^{2} \omega^{2}}{2 g}+z_{0}-\frac{\omega^{2} R^{2}}{6 g}
$$

where $(z, r)$ are cylindrical coordinates, $\omega$ is the rotation speed, $R$ the radius of the tank, and $z_{0}$ the $z$ coordinate of the free surface when the tank is at rest. When using the assumption made by Monaghan [22] about the speed of sound, the free surface is below what was expected (see Fig. 2 left).

A speed of sound closer to reality avoids compressibility inconsistency. However, the time steps are much smaller and the computation time is greatly increased. For the example of Fig. 2, the computation time was multiplied by 24 when taking $c_{0}=1480 \mathrm{~m} / \mathrm{s}$.
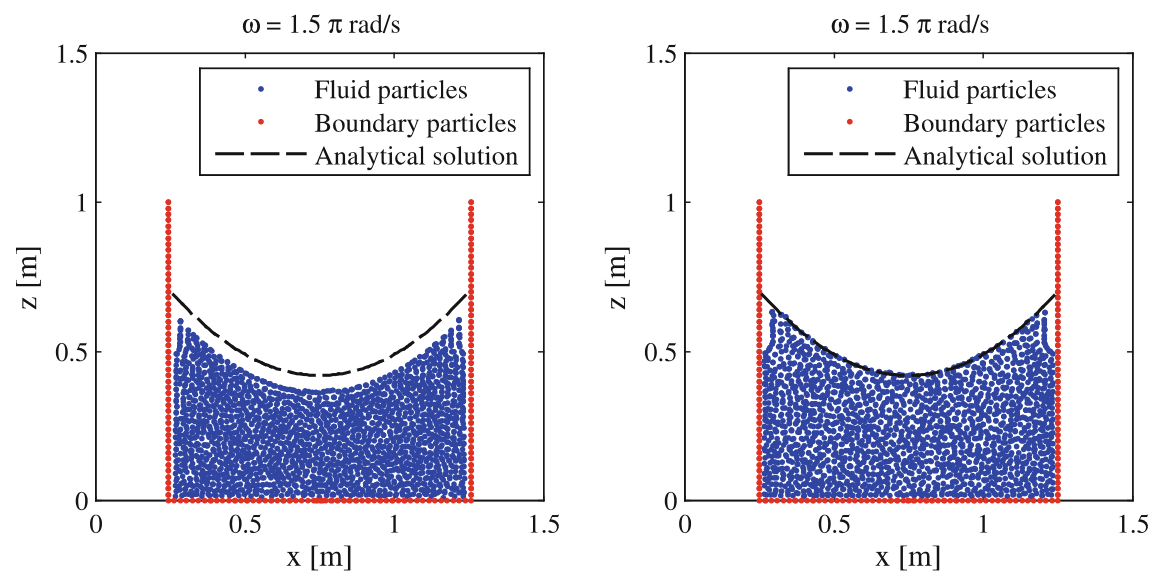

Fig. 2 On the left-hand side, $c_{0}$ is taken at $50 \mathrm{~m} / \mathrm{s}$ which leads to an over compressibility. On the right-hand side, $c_{0}$ is equal to $1480 \mathrm{~m} / \mathrm{s}$ as in reality, which leads to good results according to theory 


\subsection{Dam Break on a Dry Bed}

A well-known test case is the dam break on a dry bed. It was performed in many articles such as in $[2,10]$. It is based on the experiments of Koshizuka and Oka [14]. They measured the position of the front as well as the shape of the wave. The position of the wave front is measured dimensionless. The initial situation can be represented in 2-D as shown in Fig. 3a.

When taking a closer look at the interface between the fluid and the boundary, two observations can be made: (1) a gap is observed when a thin layer of fluid flows on a horizontal boundary (Fig. 3b) and (2) some particles stay attached to the walls of the domain (Fig. 3c). When a particle enters in the support domain of a boundary particle, a repulsive force is created which creates a gap equal to the support radius. When particles are moving away from each other, the artificial viscosity creates an attracting force between them. If this attracting force is greater than the gravity, some particles stay attached to the boundary.

The results of a numerical simulation can be compared to Koshizuka and Oka's experiment. Figure 4 gives this comparison. In order to avoid particles attached to the boundaries and consequently undesired attracting forces, a symmetry condition can be used. The effect of a symmetry condition for low number of particles is also plotted in Fig. 4. As it can be observed, the results are improved.

Recent works on the viscosity treatment [27] and the boundary conditions [5] may lead to better results.

The accuracies of time integration schemes used for this work are compared in Fig. 5. Concerning the execution time, the RK22 schemes are only $21 \%$ slower than the Euler scheme. This is due to the fact that only the distance between neighbors is re-evaluated for the correcting step. The search of neighbors is performed only for the main step.
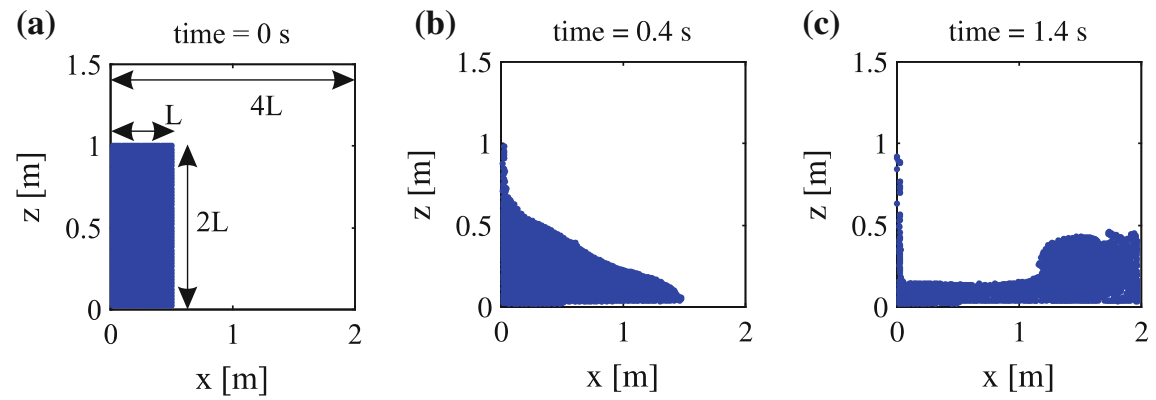

Fig. 3 The initial geometry that is plotted in figure a can be improved by doubling the size of the domain according to $x$ in order to add a symmetry condition (and a symmetric dam break). A thin gap between the fluid and the boundary can be observed in figure (b). On figures b and c, some particles stay attached to the left wall. These behaviors can be explained, thanks to the repulsive and attracting forces that are generated by the dynamic particles 


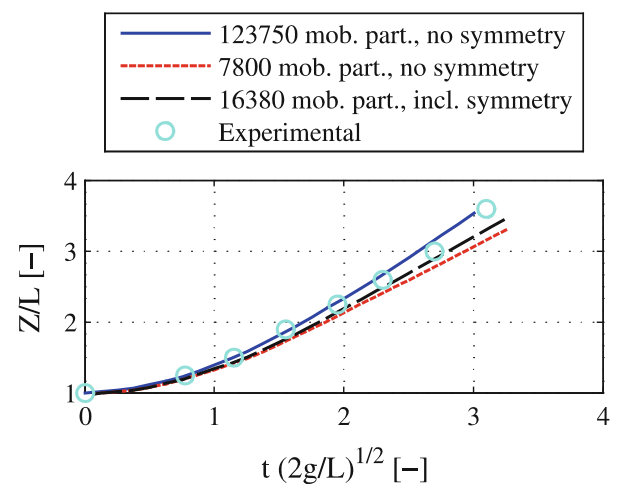

Fig. 4 Dimensionless comparison between experimental and numerical results. $Z$ is the position of the front regarding the left boundary. The numerical results match well with the experimental ones. Better results are obtained with a larger amount of particles. However, for low numbers of particles, good results can be obtained when considering a symmetry condition
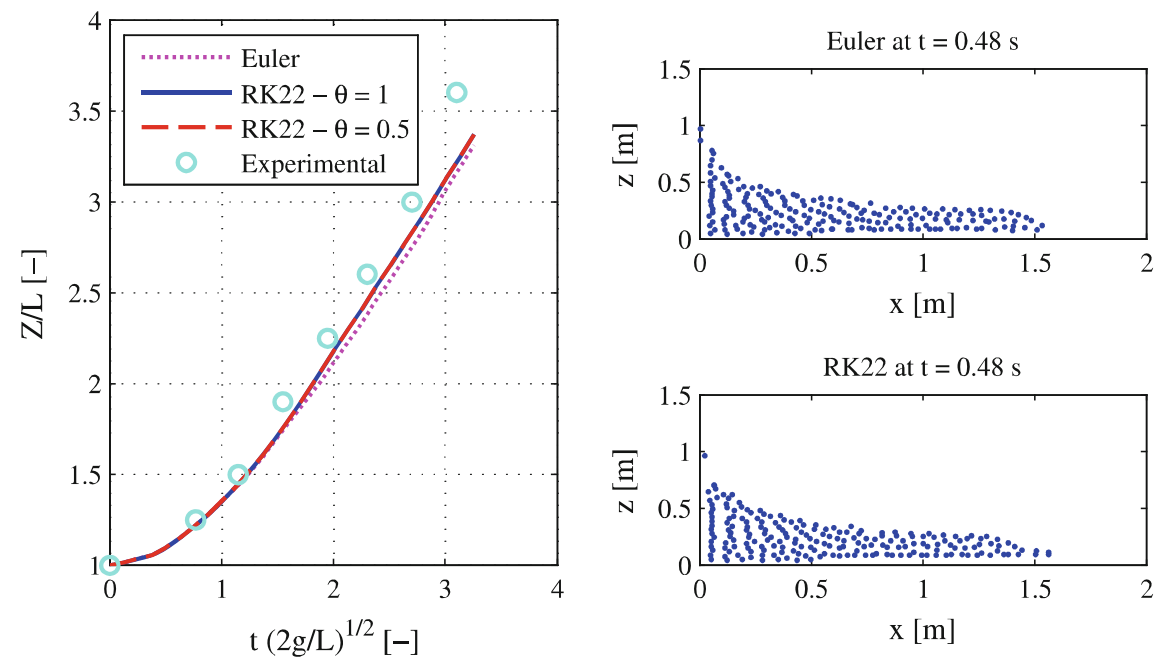

Fig. 5 The RK22 integration scheme gives better results than the Euler scheme

\subsection{Flow Over a Spillway}

This section will compare results of a steady flow over a spillway from a physical model to SPH results. In order to this, open boundaries must be implemented. These boundary conditions allow inflow and outflow in specific regions of the domain. Information about implementing this kind of boundaries can be found in [15]. When implemented, an inflow can be imposed upstream and particles are deleted when they leave the domain downstream. Initially, the free surface is set at the 
Fig. 6 Position of boundary particles and geometry of the spillway crest according to WES standard

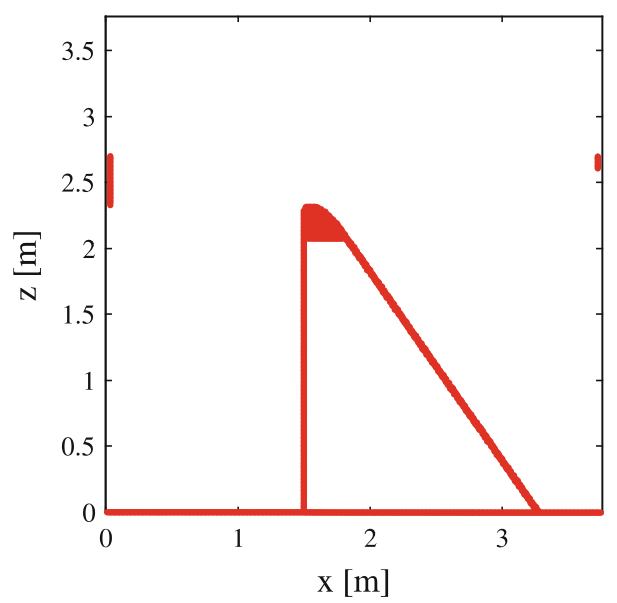

height of the crest. The flow is considered steady when the head is stabilized upstream. The shape of the spillway crest is designed according to the WES standard. In order to avoid effects from the bottom of the domain, the crest is positioned at $2.32 \mathrm{~m}$ from the bottom. The numerical model has the same dimensions as the physical model in lab (see Fig. 6). A comparison between the speed profiles will be done for an inflow of $125 \mathrm{l} / \mathrm{s}$ per meter of crest. This inflow is the design discharge of the spillway.

A reduction of section at the top of the crest can be observed (see Fig. 7). This effect is due to high repulsive forces coming from the interaction with boundary particles.

According to experimental measurements of velocity (using LS-PIV), numerical and experimental velocity profiles over the crest are compared in Fig. 8. A velocity profile in SPH can be obtained by considering a zone that extends a little to the left and to the right of the considered section. It can be seen in Fig. 8 that before the crest $\left(x / H_{d}<0\right)$ the speeds are close to experimental measurements but after the crest $\left(x / H_{d} \geq 0\right)$ the results are not conforming to experimental measures. This

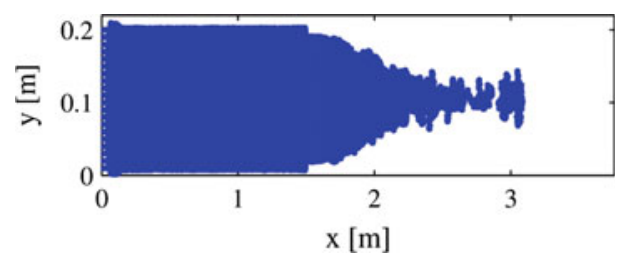

Fig. 7 When the water overtops the spillway, the lateral boundary particles exert a repulsive force on the fluid particles which repels them from the borders 

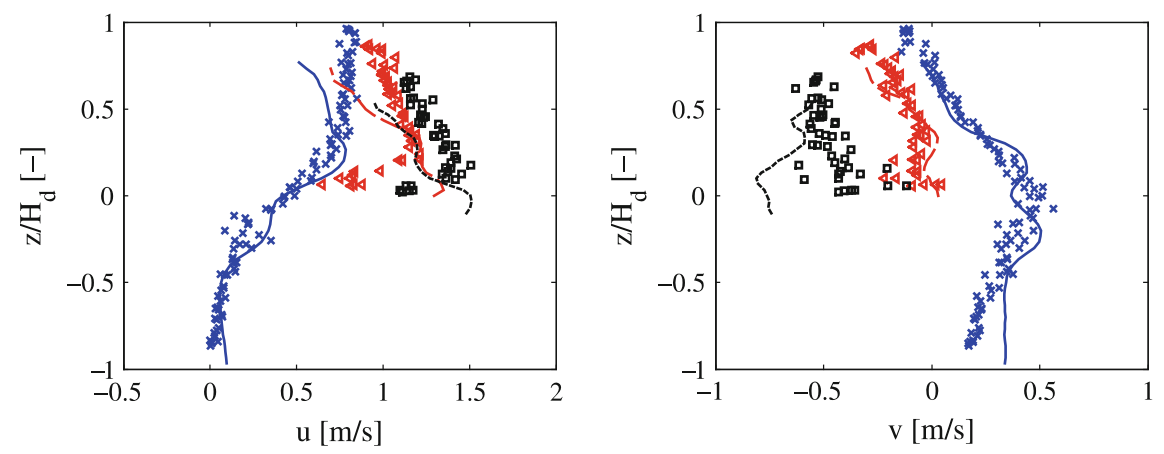

Fig. 8 Horizontal (u) and vertical (v) speed profiles at $x / H_{d}=-0.5$ (plain line and crosses), $x / H_{d}=O$ (dashed line and triangles), and $x / H_{d}=0.5$ (dotted line and squares). Lines are for experimental measures and shapes for numerical results

behavior can be explained by (a) too important viscous effects on the spillway, (b) the behavior of the dynamic particles which create too strong repulsive forces, and (c) the reduction of section at the crest due to the boundary particles. Once more, better boundary conditions and a more appropriate viscous term would lead to more quantitative results.

\subsection{Shape of Flow Over a Weir}

Another test case can consist in comparing the shape of a nape of flow over a weir to an analytical profile. This analytical profile can be digitalized from [11]. The resulting polynomials are given by (11) and (12) for, respectively, the upper nape and the lower nape.

$$
\begin{array}{r}
\frac{z}{H}=-0.04\left(\frac{x}{H}\right)^{3}-0.2\left(\frac{x}{H}\right)^{2}-0.26\left(\frac{x}{H}\right)+0.88-1 \leq \frac{x}{H} \leq 2 \\
\frac{z}{H}=-0.33\left(\frac{x}{H}\right)^{4}+1.18\left(\frac{x}{H}\right)^{3}-1.90\left(\frac{x}{H}\right)^{2}+0.84\left(\frac{x}{H}\right) 0 \leq \frac{x}{H} \leq 1.5
\end{array}
$$

In order to avoid effects from the bottom of the domain, we considered a weir's height of $2.3 \mathrm{~m}$. The theoretical profiles and the one obtained numerically are compared in Fig. 9. Due to viscous tensions, these two different approaches do not fit well. There is also an influence from the boundary particles on the section of flow over the weir as it was observed in the previous test case (Fig. 7). 
Fig. 9 The SPH does not fit so well with the analytical profiles

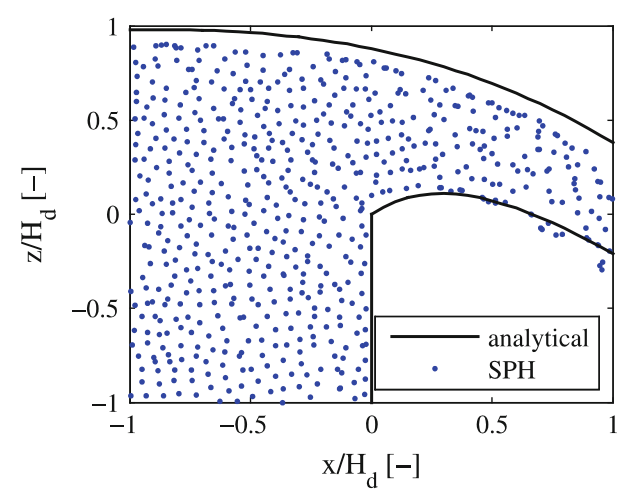

\section{Conclusion}

Even if the SPH method was introduced more than 35 years ago, it is still under large developments. However, a simple model can be obtained quickly. This paper is based on a master thesis in which a 3-D SPH code was implemented [7]. The goal of this paper was to present some test cases useful to validate a program. According to our experience, we pointed out some undesired effects that are not well referenced yet in the literature. Recent developments introduce new possibilities to deal with the undesired effects shown in this paper.

The still tank test case showed that an initial equilibrium state is impossible in some situations. The next test case concerned a spinning tank. It showed that the usual assumption of a low speed of sound can lead to an overcompressibility. In order to avoid this, a pressure Poisson equation is one of the possibilities [16]. The dam break test case pointed that the dynamic boundary particles can cause undesired effects such as attracting forces. Finally, the flows over a spillway and a weir emphasized the fact that boundaries have a large effect on SPH simulation. We are currently working on a 2-D code that should improve the situation [19]. However, boundary conditions are still a difficulty in SPH.

Aknowledgments The authors are grateful for the data provided and the fruitful discussions with Dr. Yann Peltier.

\section{References}

1. Canor, T., \& Denoël, V. (2013). Transient Fokker-Planck-Kolmogorov equation solved with smoothed particle hydrodynamics method. International Journal for Numerical Methods in Engineering, 94(6), 535-553.

2. Crespo, A. J. C., Gómez-Gesteira, M., \& Dalrymple, R. A. (2007). Boundary conditions generated by dynamic particles in SPH methods. Computers, Materials and Continua, 5(3), $173-184$. 
3. Dalrymple, R. A., \& Knio, O. (2001). SPH modelling of water waves. Sweden: ASCE.

4. Dymond, J. H., \& Malhotra, R. (1988). The Tait equation: 100 years on. International Journal of Thermophysics, 9(6), 941-951.

5. Ferrand, M., et al. (2013). Unified semi-analytical wall boundary conditions for inviscid, laminar or turbulent flows in the meshless SPH method. International Journal for Numerical Methods in Fluids, 71(4), 446-472.

6. Gingold, R. A., \& Monaghan, J. J. (1977). Smoothed particle hydrodynamics-theory and application to non-spherical stars. Monthly Notices of the Royal Astronomical Society, 181, $375-389$.

7. Goffin, L. (2013). Development of a didactic SPH model. Liege: University of Liege (ULg).

8. Gomez-Gesteira, M., et al. (2012). SPHysics - development of a free-surface fluid solverPart 2: Efficiency and test cases. Computers and Geosciences, 48, 300-307.

9. Gomez-Gesteira, M., et al. (2012). SPHysics - development of a free-surface fluid solverPart 1: Theory and formulations. Computers and Geosciences, 48, 289-299.

10. Gomez-Gesteira, M., et al. (2010). State-of-the-art of classical SPH for free-surface flows. Journal of Hydraulic Research, 48(1), 6-27.

11. Hager, W., \& Schleiss, A. (2009). Traité de Génie Civil, Volume 15-Constructions Hydrauliques-Ecoulements Stationnaires. Switzerland: PPUR-Presses Polytechniques Romandes.

12. Jánosi, I. M., et al. (2004). Turbulent drag reduction in dam-break flows. Experiments in Fluids, 37(2), 219-229.

13. Johnson, G. R., Stryk, R. A., \& Beissel, S. R. (1996). SPH for high velocity impact computations. Computer Methods in Applied Mechanics and Engineering, 139(1), 347-373.

14. Koshizuka, S., \& Oka, Y. (1996). Moving-particle semi-implicit method for fragmentation of incompressible fluid. Nuclear Science and Engineering, 123(3), 421-434.

15. Lastiwka, M., Basa, M., \& Quinlan, N. J. (2009). Permeable and non-reflecting boundary conditions in SPH. International Journal for Numerical Methods in Fluids, 61(7), 709-724.

16. Lind, S. J., et al. (2012). Incompressible smoothed particle hydrodynamics for free-surface flows: A generalised diffusion-based algorithm for stability and validations for impulsive flows and propagating waves. Journal of Computational Physics, 231(4), 1499-1523.

17. Liu, G. (2003). Mesh free methods: moving beyond the finite element method. New York: CRC Press.

18. Liu, G. G.-R., \& Liu, M. (2003). Smoothed particle hydrodynamics: a meshfree particle method. Singapore: World Scientific.

19. Lodomez, M. (2014). Determining the characteristics of a free jet in 2-D by the SPH method. Liege: University of Liege (ULg).

20. Lucy, L. B. (1977). A numerical approach to the testing of the fission hypothesis. The astronomical journal, 82, 1013-1024.

21. Monaghan, J. J. (1988). An introduction to SPH. Computer Physics Communications, 48(1), $89-96$.

22. Monaghan, J. J. (1994). Simulating Free Surface Flows with SPH. Journal of Computational Physics, 110(2), 399-406.

23. Monaghan, J. J., \& Kos, A. (1999). Solitary waves on a cretan beach. Journal of Waterway, Port, Coastal and Ocean Engineering, 125(3), 145-154.

24. Monaghan, J. J., \& Lattanzio, J. C. (1985). A refined particle method for astrophysical problems. Astronomy and Astrophysics, 149, 135-143.

25. Nelson, R. P., \& Papaloizou, J. C. (1994). Variable smoothing lengths and energy conservation in smoothed particle hydrodynamics. arXiv preprint astro-ph/9406053.

26. Randles, P. W., \& Libersky, L. D. (1996). Smoothed particle hydrodynamics: Some recent improvements and applications. Computer Methods in Applied Mechanics and Engineering, 139(1-4), 375-408. 
27. Violeau, D. (2009). Dissipative forces for Lagrangian models in computational fluid dynamics and application to smoothed-particle hydrodynamics. Physical Review E-Statistical, Nonlinear, and Soft Matter Physics, 80(3).

28. Violeau, D. (2012). Fluid Mechanics and the SPH method: theory and applications. Oxford: Oxford University Press.

29. Violeau, D., \& Leroy, A. (2014). On the maximum time step in weakly compressible SPH. Journal of Computational Physics, 256, 388-415. 